\title{
Monográfico: Perspectivas Actuales de Evaluación e Intervención en Atención Temprana
}

\section{Editorial}

\author{
Coordinadores: Rita Pilar Romero-Galisteo ${ }^{1}$, Carmen Barajas ${ }^{2}$ y Pablo Gálvez-Ruiz ${ }^{3}$
}

\begin{abstract}
${ }^{1}$ Centro de Atención Temprana del Excmo. Ayto. de Antequera (Málaga). Facultad Ciencias de la Salud. Departamento de Fisioterapia. Universidad de Málaga, España. ${ }^{2}$ Facultad de Psicología. Departamento de Psicología Evolutiva y de la Educación, Universidad de Málaga, España. ${ }^{3}$ Facultad de Educación. Departamento de Educación en Internet. Universidad Internacional de La Rioja, España.
\end{abstract}

Disponible online 31 de agosto de 2015

Tradicionalmente la Atención Temprana (AT) ha sido un ámbito de estudio que ha visto incrementado el interés científico tanto en Europa como en Norteamérica, no siendo distinto en España, donde algunos investigadores ofrecen interesantes resultados para mejorar tanto la evaluación como la intervención en la práctica diaria.

Actualmente se utiliza el concepto de AT, que sustituye al originario de "estimulación precoz", que es como inicialmente se llamó a este ámbito de intervención (Robles-Bello y Sánchez-Teruel, 2013). El Grupo de Atención Temprana (2005) la define como:

El conjunto de intervenciones dirigidas a la población infantil de 0-6 años, a la familia y al entorno, que tienen por objetivo dar respuesta lo más pronto posible a las necesidades transitorias o permanentes que presentan los niños con trastornos en su desarrollo o que tiene el riesgo de padecerlos (p. 13)

La AT pasa actualmente por un momento controvertido en nuestro país. En tanto que algunos acuerdos legislativos alcanzados recientemente pueden llegar a entorpecer la implementación de una intervención de calidad, los profesionales e investigadores expertos en este campo arrojan nuevas perspectivas que facilitan el desarrollo de la misma.

Importantes documentos publicados en los últimos 30 años muestran la evolución del concepto de AT, en el que la salud, la educación y las ciencias sociales (especialmente la psicología) están directamente implicadas (European Agency for Development in Special Needs Eduation, 2005). Se ha pasado de un modelo centrado en el niño a un tipo de intervención en la que participan de forma activa el niño, la familia y el entorno y, por ende, mayor número y diversidad de profesionales.

En este contexto, la revista Escritos de Psicología lanza este monográfico con el que pretende unir investigación y práctica clínica, acercar a investigadores y a profesionales que atienden directamente a niños y familias, recoger trabajos que aborden la AT desde las diferentes perspectivas implicadas en ella. En definitiva, el objetivo de este número es avanzar en el estudio de la AT aunando esfuerzos y aportando novedades en cuanto a técnicas de intervención y herramientas de evaluación específicas.

Resulta imprescindible fusionar iniciativas políticas con las actuales perspectivas que sobre el desarrollo se han ido publicando en los últimos años (Guralnick, 2013; Shonkoff \& Phillips, 2000). Sobre este tema versa la aportación que desde la Universidad de Washington nos remite el profesor Guralnick, titulada: "Merging Policy Initiatives and Developmental Perspectives in Early Intervention" (Uniendo iniciativas políticas y perspectivas de desarrollo en Atención Temprana). En este artículo se proponen estrategias para mejorar la eficacia de los servicios de AT en la comunidad que distintos autores han ido desarrollando en el marco de los patrones de desarrollo. A pesar de que la ciencia ha indicado ya los beneficios de la AT, la implementación de la misma en el marco del desarrollo evolutivo, incluso en países avanzados, dista de ser completa. El manuscrito sugiere la interacción de iniciativas políticas y principios del desarrollo como única manera de mejorar el bienestar de los niños vulnerables y sus familias. En consecuencia, como el propio autor concluye, la perspectiva del desarrollo puede y debe combinarse con patrones de interacción familiar cuando se establezcan iniciativas políticas para mejorar los servicios de AT.

Reivindicar el papel preventivo de la AT supone atender a los factores de riesgo biológico que puedan presentarse desde el momento mismo de la concepción. Dos artículos presentan revisiones teóricas interesantes en esta línea. El manuscrito que envían Roncallo, Sánchez de Miguel y Arranz Freijo lleva por título "Vínculo materno-fetal: implicaciones en el desarrollo psicológico y propuesta de intervención en Atención Tem- 
prana". En él, los autores plantean una interesante intervención que incluya el período prenatal basándose en la evidencia de que el estado psicológico de la madre influye en el feto. El objetivo es doble, por un lado potenciar las prácticas de parentalidad positiva y, por otro, favorecer la salud materno-infantil. De esta manera, afirman, el vínculo materno-fetal constituye un factor protector del vínculo madre-bebé y, por tanto, del desarrollo psicológico posterior del recién nacido.

El trabajo presentado por Escolano, cuyo título es "Discordancia del peso al nacer: consecuencias y su perdurabilidad en el desarrollo psicológico del gemelo de menor peso intrapar", parte del incremento de nacimientos de gemelos con discordancia de peso. Al suponer el bajo peso un factor de riesgo en el desarrollo, que a su vez incrementa la tasa de mortalidad y morbilidad, los menores que presentan esta condición son atendidos por los equipos de AT. La autora concluye que las diferencias en el desarrollo psicológico perduran hasta la adolescencia y juventud, siendo extensas tanto las dimensiones afectadas como su perdurabilidad.

Los modelos contemporáneos de AT sitúan a la familia como pilar fundamental en el que se apoya la intervención. En esta línea Martínez Moreno y Calet presentan en el trabajo "Intervención en Atención Temprana: Enfoque desde el ámbito familiar" una revisión teórica sobre la evolución de la AT y los modelos de intervención. En él se recoge la importancia del entorno familiar en los programas de atención durante los primeros años de vida, destacando la consolidación del interés científico de esta disciplina.

Dos informes de investigación aportan datos de interés sobre la AT desde el ámbito familiar, el artículo firmado por Fernández Alcántara, Cruz Quintanilla, Toral López, González Carrión, Rivas Campos y Pérez Marfil, titulado "Efectos de un programa de alta precoz sobre las preocupaciones de los padres de niños prematuros", se centra en la importancia de la exploración de las experiencias y cambios en la dinámica familiar tras el alta hospitalaria de niños prematuros, teniendo en cuenta el gran impacto emocional que produce en los padres. Utilizan una metodología cualitativa para analizar entrevistas semiestructuradas, obteniendo como resultado la existencia de importantes preocupaciones relacionadas con las habilidades y el cuidado del hijo; los padres y madres destacaron sentirse satisfechos con la implantación del programa de alta precoz, al apreciar importantes beneficios emocionales y repercusiones positivas en el cuidado y la relación con sus hijos.

Por su parte, el informe de investigación de Mayorga Fernández, Madrid Vivar y García Martínez, “Aprender a trabajar con las familias en Atención Temprana: estudio de caso", se centra en la identificación de las necesidades de las familias que acuden a un Centro de Atención Infantil Temprana (CAIT) generalista. Para ello administran a una muestra de 58 familias un cuestionario de satisfacción elaborado por los propios autores, basado en indicadores de capacitación familiar identificados en estudios previos. Los resultados indican que las familias, independientemente de si tienen uno o más de un miembro con discapacidad, muestran interés por participar en programas de apoyo para y entre familias, que podrían implantarse desde los CAITs, en los que se les proporcione información, formación y asesoramiento, así como la oportunidad de apoyo emocional y comunicación con otras familias con experiencia en atención a personas con necesidades especiales.

La formación de los profesionales de la AT, así como la colaboración entre éstos y las familias, es otro ámbito que está generando actualmente líneas de investigación y de actuación. El estudio que se presenta en el informe de investigación de Estévez Casellas, Castello Aracil y Tirado, “Conocen los profesionales las necesidades de los padres de niños con patología del desarrollo?", tiene como objetivo identificar y comparar las necesidades que manifiestan tener padres y profesionales que atienden a niños con patologías del desarrollo; para ello aplican sendos cuestionarios de elaboración propia a una muestra de 37 familias y 20 profesionales. Los resultados indican que, aunque padres y profesionales coinciden en las necesidades que muestran, difieren en la valoración de la importancia que les otorgan.

La investigación que presentan Romero Galisteo, Gálvez Ruiz, Belda Oriola y Millá Romero, "Percepción de la calidad en Centros de Atención Temprana: Resultados de un análisis comparativo", aporta datos de dos estudios sobre la calidad percibida, en una muestra de 397 usuarios, de dos Centros de Desarrollo Infantil y Atención Temprana (CDIAT). En cada uno de los dos estudios (separados por un intervalo de dos años y dos meses) se administró en ambos centros el Inventario de Calidad en Centros de Atención Infantil Temprana. En ambos estudios los aspectos del servicio mejor valorados por los usuarios fueron los referidos al personal especializado y a la información técnica; además, en uno de los dos CDIAT la valoración que hicieron los usuarios de las instalaciones y de las salas de tratamiento y material mejoró diferencialmente del primer al segundo estudio, lo que demuestra la utilidad de la información que proporciona este instrumento para la implementación de procesos de mejora continua.

En definitiva, la selección de trabajos que conforman este monográfico es un reflejo de la realidad interdisciplinar, académica y aplicada, que caracteriza a la AT y pone de manifiesto la necesidad de seguir avanzando en trasladar la evidencia científica a la práctica clínica.

\section{Referencias}

1. Grupo de Atención Temprana [GAT]. (2 $2^{\mathrm{a}}$ Ed.). (2005). Libro blanco de la Atención Temprana. Madrid: Real Patronato de Prevención y de Atención a Personas con Minusvalía.

2. Guralnick, M.J. (2013). Developmental science and preventive interventions form children at environmental risk. Infants \& Young Children, 26, 270-285. doi: 10.1097/IYC. ob.13e3182a68f

3. Robles-Bello, M.A. y Sánchez-Teruel, D.(2013). Atención infantil temprana en España. Papeles del Psicólogo, 34, 132-143. 
EDITORIAL

4. Soriano, V. (Ed.) (2005). Early Childhood Intervention. Analysis of Situations in Europe: Key aspects and recommendations (summary report). Brussels: European Agency for Development in Special Needs Education. Consultado en Junio 2015, disponible en http://www.european-agency. org/publications/ereports/early-childhood-intervention/ eci en.pdf.

5. Shonkoff, J.P. y Phillips, D.A. (2000). From neurons neighbourhoods: The science of early child development. Committee on Integrating the Science of Early Childhood Development. Washington D.C: National Academy Press. 